ISSN 1409-2441
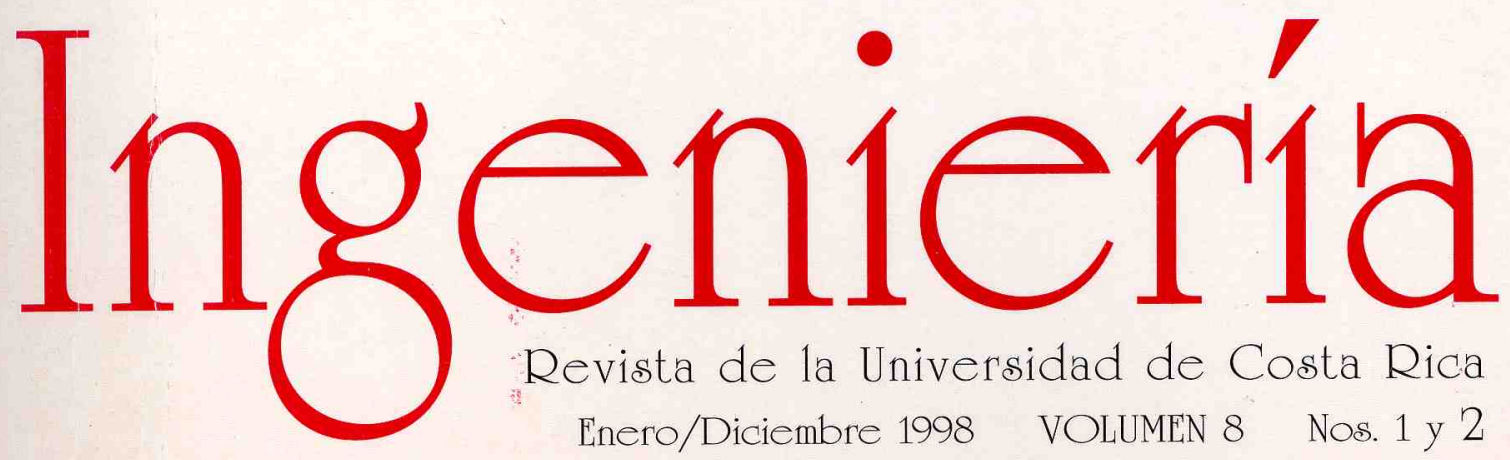

Revista de la Universidad de Costa Rica Enero/Diciembre 1998 VOLUMEN 8 Nos. 1 y 2 


\title{
METODOANALITICO PARA CALCULAR EL FACTOR GEOMETRICO J DE ENGRANES RECTOS ESTANDARIZADOS
}

\author{
Alejandro PachecoM 1
}

\section{Resumen}

En e;(e artículo se presenta uu método analítico para calcular el factor geométrico J para el diseño de engranes rectos como una rentajosa ahemativa al método gráfico estandarizado de la AGMA. El método desalTollado produce resultados que aproximan fielmentea los valores AGMA para el caso de engranes fabricados con cortadores de generación y a la vez permite generar I'aloresdel factor J, ha,ta ahora no di,ponibles, para el disello p3lticular de engranes fabricados con cortadores de conformación.

\section{Summary}

Ul this paper, an analytical melhod for calculating Ihe geometry factor $\mathrm{J} 01$ ' spur gears is presented as an altemative to the graphical mcthod of the AGMA standards. 111e anal)tical method produces $\mathrm{J}$ values that approach closely the AG\}.\{A values for gears made by generating Clrtters, and additionally allows calculating $\mathrm{J}$ values for the partieular design of gears made by fonneutlers

\section{INTRODUCCION}

En este artículo se presenta tUI modelo matemáticopara calcular el llamado factor $\mathrm{J}$ de un engrane recto estandarizado. Los métodos clásicosy tradicionales para diseílar este tipo de engranes se basan en un procedimiento establecido por la American Gear Manufadurers Association (AGMA), donde, el factor de forma $\mathrm{J}$ se determina por medio de gráficas publicadas y patentadas por la misma AGMA.

Es importante aclarar que el método presentado en este artíCulo se basa en una revisión y refoffimlación extensivas del modelo matemáticopara calcular el factor $J$ en engranes rectos. publicado por el autor previamente en estarevista ( Pacheco. A .y Rodríguez, A, Vol. 6. No. 1, 1996). El modelo actual contiene mejorasde forma y de fondo. la nomenclatura y la simbología gráfica fueron simplificadas, se eliminó el proceso nunlérico iterativo para localizar la sección critica, el esfuerzo de flexión se calcula con una ecuación más exacta y se obtienen los radios de filete en la raíz del diente en fonna diferenciada para engranes cortados por generación o confonnación. En consecuencia, los nuevos resultados son más exactos y además permiten calcular valores del factor J exclusivamente para el diseílo de engranes cortados con herramientas de conformación, lo cual se considera un aporte novedoso, ya que esta infonnación no está contenida en los estándares de la AGMA, ni se encuentra publicada en la literatura disponible.

\section{DEFINICIÓN DEL FACTOR GEOMÉTRICO $\boldsymbol{J}$}

Como primer paso para la fommlación del modelo matemático del factor $\mathrm{J}$, se requiere una clara identificación gráfica de los parámetros geométricos que estarán involucrados en el mismo. Para ello, considérese la figura NO.1 que muestra 
esquemáticamente tuI diente de engrane recto con perfil de evolvente y para el cual se define la siguiente simbología:

O: centro del engrane.

$H$ : $\quad$ punto más alto de contacto.

$P$ : $\quad$ punto de paso sobre el perfil del diente

$B: \quad$ punto inicial de la evolvente.

C: punto sobre la curva de enlace en la raíz del diente que corresponde a la sección critica.

G: centroide del área critica a la flexión en la base del diente.

GC: plano que define a la sección crítica del diente.

Q: $\quad$ punto de intersección entre la línea de acción de la carga y la línea o eje central del diente.

E: centro instantáneo de la generatriz de la evolvente en el punto de contacto.

$W$ : carga normal al diente.

( $Y$ : esfuerzo de flexión en la sección crítica. $t: \quad$ anchura basal del diente en la sección crítica.

h: brazo de palanca de la carga $\mathrm{W}$ con respecto al centroide $\mathrm{G}$ del área crítica a la flexión.

a: $\quad$ adendo

b: $\quad$ dedendo

$r_{r \prime}: \quad$ radio del Círculo de adendo.

$r_{P^{\prime}} \cdot \quad$ radio del Círculo de paso.

$r_{b}: \quad$ radio del CÍrculo base

$\theta$ : ángulo de presión de la línea de transmisión con respecto al eje central del diente. Se le llamará ángulo de presión relativo.

$\theta_{H}$ : valor del ángulo $\quad e$ cuando la carga se aplica en el punto más alto de contacto $\mathrm{H}$.

$F$ : ancho de cara del diente (no mostrado)

rp: ángulo de presión nominal (no mostrado)

Para calcular el esfuerzo flector $(Y$ se recurre a la hipótesis de que el diente actúa como una viga empotrada sometida a flexión y comprensión en el área de unión con el cuerpo del engrane. Así:

$\sigma=\left\{\left(\frac{M c}{I}\right)-\frac{W r}{A}\right\} K_{f}$

donde:

$M=I r O Q \times W \mid=h \cdot$ Wsene; $\mathrm{e}=t / 2 ; 1=\frac{F t^{3}}{12} \quad K f=$ factor de concentración de esfuerzos por fatiga; $W_{r}=W$ cose $; A=F t$

Sustituyendo términos en la ecuación No. 1: 
${ }_{j}=K_{f} \frac{W_{\text {cose }}}{F}\left\{\frac{6 h \text { tane }}{t^{2}} \cdot{ }_{t}^{\prime}\right\}$

La carga nonnal $W$ puede relacionarse con la carga transmitida nominal $\sim$ de acuerdo con la relación:

$W=\left(\frac{W_{t}}{\cos \phi}\right) m_{N}$

donde: $m_{N}=$ relación de compartición de carga.

Sustituyendo la ecuación NO.3 en la NO.2:

$\sigma=K_{f} \frac{W_{t} \cos \theta}{F \cos \phi} m_{N}\left(\frac{6 h}{t^{2}} \tan \theta-\frac{1}{t}\right)$

Esta relación puede arreglarse de la manera siguiente:

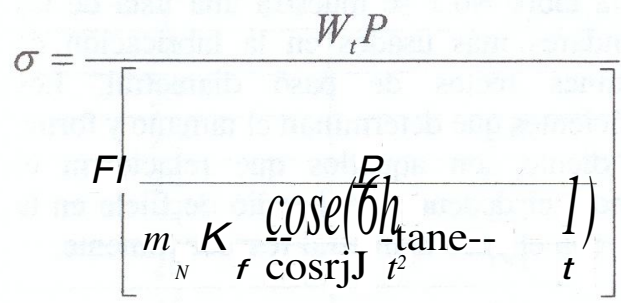

donde: $P=$ paso diametral.

Haciendo una analogía entre esta ecuación y la fónnula clásica de Lewis, se define el factor de fonna $J$ para el diente del engrane, como:

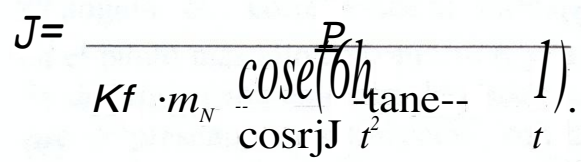

Así el esfuerzo flector ( $J$ genérico puede. expresarse según la relación más compacta:

$C J=\frac{W_{t} \cdot p}{F J}$ 


\section{FACTOR GEOMÉTRICO $J$ DE LA AG}

En un diente de engrane recto el esfuerzo flector máximo ocurre cuando la carga se aplica en el punto más alto de contacto. En esta posición, el ángulo $B$ (ver figura No.l) adquiere el valor $B_{H} \quad \mathrm{Y}$ el punto de contacto coincide con el punto más alto de contacto que se denominará como punto $\mathrm{H}$. Por lo tanto, el factor de forma $J$ de la AGMA se define como $J=J(B=B H)$, o bien:

$J=K_{f} \cdot m_{H} \frac{\cos B H}{\cos r />} P\left(h_{2} \operatorname{tanBH}-t\right)$

De aquí en adelante el problema de calcular $J$ consfstirá en obtener los valores de todos los parámetros representados en la ecuación No.7

\section{CARÁCTER ADIMENSIONAL DEL FACTORJ.}

Un análisis de las dimensiones del factor $J$ expresado según la ecuación NO.7 revela que éste es un parámetro adimensional. Aún más, sabiendo que para un engrane nonnalizado con dientes de evolvente, todas las dimensiones del diente son directamente proporcionales a IIP, es fácil concluir que el factor $J$ no solo será adimensional sino que será independiente del valor de $P$.

\section{PARÁMETROS BÁSICOS INDEPENDIENTES}

Siendo $J$ un factor puramente geométrico, su valor en última instancia dependerá de los siguientes parámetros básicos que definen la geometría de cualquier sistema de engranes nonnalízados:

- Número de dientes del engrane bajo estudio $N$.

- NÚmero de dientes del engrange compaIlero $\mathrm{Ne}$

- Angulo de presión nominal $\mid>$.

- Sistema estandarizado del tamaño del diente.

\section{VALOR DE PASO DIAMETRAL P}

Por conveniencia en los procesos de cálculo involucrados en el modelo matemático, se trabajará con un paso diametral unitario, es decir $P=$ ldiente $/$ pul.

\section{SISTEMAS ESTANDARIZADOS DIENTES. 7}

$\mathrm{DE}$

En la tabla No.l se muestra lila ysta de los estándares más usados en la fabricación de engranes rectos de paso diametral. Los coeficientes que detenninan el tamallo y forma del diente son aquellos que relacionan el adendo, el dedendo, y el radio de filete en la raíz con el paso diametral respectivamente.

En el caso de engranes cortados con fresa madre o cualquier otra herramienta que produzca dientes de generación, las dimensiones fundamentales del diente se obtienen con las fónnulas siguientes:

$$
\begin{aligned}
& a=\frac{K_{0}}{P} \\
& b=\frac{K b}{P} \\
& r_{t}=\frac{K r}{P}
\end{aligned}
$$


$r,=r,+\frac{(b-r, y}{r p+b-r,}$

El factor $r t$ es el radio de curvatura de la punta del cortador de generación y $r($ es el radio de filete aproximado en la raíz del diente del engrane producido [Peterson, 1974]. Este valor de $r f$ se dice que es aproximado porque en los engranes de generación. la curva de enlace en la raíz del diente es una curva trocoidal, más parecida a una porción de elipse que a un círculo.

Las ecuaciones No. 8, 9, Y 10 son también válidas para engranes cortados con fresas de conformación. Para este caso, la curva de enlace en la raíz del diente coincide con la forma exacta de la punta del cortador, la cual posee un borde circular cuyo radio está dado por la relación estandarizada siguiente (Casillas, AL., 1988) :

$r_{f}=r,=\frac{0.3}{P}$

Es importante sella lar que las relaciones anteriores son válidas también para los engranes estandarizados del sistema de módulo , con solo sustituir el factor $1 / P$ por el módulo $n I$.

\begin{tabular}{|c|c|c|c|}
\hline $\begin{array}{c}\text { Angulo de presión } \\
\boldsymbol{\phi}\left({ }^{\circ}\right)\end{array}$ & $\begin{array}{c}\text { Constante de adendo } \\
\mathbf{K a}\end{array}$ & $\begin{array}{c}\text { Constante de } \\
\text { Dedendo } \\
\mathbf{K b}\end{array}$ & $\begin{array}{c}\text { Constante de radio de la punta } \\
\text { del cortador Kr }\end{array}$ \\
\hline 20 & 1.0 & 1.2500 & 0.3000 \\
\hline 20 & 1.0 & 1.4000 & 0.3500 \\
\hline 20 & 1.0 & 1.1570 & 0.2390 \\
\hline 20 & 0.8 & 1.0000 & 0.3040 \\
\hline 25 & 1.0 & 1.2500 & 0.3000 \\
\hline 25 & 1.0 & 1.3500 & 0.2447 \\
\hline 25 & 1.0 & 1.3154 & 0.2700 \\
\hline
\end{tabular}

TABLA No.1. Sistemas estandarizados de dientes de uso común para engranes rectos cortados por generación

\section{DETERMINACIÓN DEL ÁNGULO eH}

El ángulo eH corresponde al fonnado entre la línea de acción de la carga $W$ cuando esta se aplica en el punto más alto de contacto $H$ y la línea central del diente, tal como se muestra en la figura NO.I. Su determinación entonces depende de ubicar precisamente dicho punto más alto de contacto, análisis que se presenta a continuación, con la ayuda de la figura No.2, en la cual se utiliza la siguiente nomenclatura complementaria:

O: centro del engrane analizado

Oc: $\quad$ centro del engrane compallero 
$P$ : $\quad$ punto de paso sobre la línea de centros

$H$ : $\quad$ punto más alto de contacto

$B$ : $\quad$ origen del perfil de evolvente sobre el círculo base

$r_{a c}: \quad$ radio de adendo del engrane compañero

$r_{p c}: \quad$ radio de paso del engrane compañero

$r_{b c}: \quad$ radio base del engrane compallero

$K$ : $\quad$ primer punto de contacto

$L \quad$ : último punto de contacto

$\mathrm{Pb}$ : $\quad$ paso circular base

$r_{H, C P}$ : ángulos involumétricos

Analízando - el triángulo OEQ, se nota que el ángulo en Q es igual a eH. por lo tanto : $\theta_{H}=90-\gamma_{H}$

A su vez, por relaciones involumétricas, el ángulo $r_{H}$ puede obtenerse como:

$r H=r j J \cdot \stackrel{90}{\underline{N}}-\stackrel{180}{-;-(\tan r j \mathrm{~J}}-\operatorname{tanCPH} \quad)$

donde, gracias al triángulo $\mathrm{OEH}$ :

$\tan \varphi_{H}=\frac{E H}{r_{b}}=\left(\frac{r_{p}}{r_{b}}+\frac{r_{p c}}{r_{b}}\right) \operatorname{sen} \phi-\sqrt{\left(\frac{r_{a c}}{r_{b}}\right)^{2}-\left(\frac{r_{b c}}{r_{b}}\right)^{2}}+\frac{p_{b}}{r_{b}}$

Expresando los diferentes radios en función de los números de dientes $N$ y $N$ e' $Y$ después de algunas transformaciones, se logra obtener:

$\tan C P H=\frac{2 r c+(1+N c)}{N}+\tan r j J-. \quad \begin{gathered}N c \\ N\end{gathered} \sqrt{\left(\frac{1+\frac{2 K_{a}}{N_{C}}}{\cos r j \mathrm{~J}}\right)^{2}-1}$ 
Ahora se combinan las ecuaciones No.13, 14 Y 16 para obtener el ángulo ()$H$ en grados de la manera siguiente:

$\theta_{H}=90-\phi-\frac{270}{N}+\frac{180 N_{C}}{\pi N}\left[\sqrt{\left(\frac{1+\frac{2 k_{a}}{N_{C}}}{\operatorname{cosrjJ}}\right)^{2}-1-t a n r j J}\right]$

Nótese que el valor de ()$H$ resultante será en grados, y que como se había establecido antes, dependerá de los parámetros fundamentales $\mathrm{N}$, $\mathrm{Ne} ' C P$, mientras que el sistema estandarizado del diente fijará el valor del coeficiente $K_{a}$.

\section{CÁlCULO DE $h, t$}

Como se puede apreciar en la figura NO.1, el cálculo de los valores de $h$ y $t$ depende de la ubicación de la sección critica, es decir aquella sección en la cual el esfuerzo ( $J$ resulte ser máximo.

Con ayuda de la figura No.3, es posible realízar un análisis matemático que interrelacione las variables $h$ y $t$, utilizando funciones geométricas de la evolvente y del arco de enlace en la raíz del diente. Con esto se logra expresar $t$ en función de $h$. Luego derivando la función de esfuerzo dada por la ecuación No.4, se puede obtener el valor de $\mathrm{h}$ que maximiza dicha función. Este procedimiento conduce a ecuaciones trascendentales, cuya solución requiere la aplicación de uno o más métodos numéricos. Este camino es el más riguroso y correcto desde un punto de vista estrictamente matemático.

Como ya se demostró anterionnente (ver ecuación NO.4); el esfuerzo de tensión en el punto C (figuras NO.1 y NO.3 ) puede expresarse como:

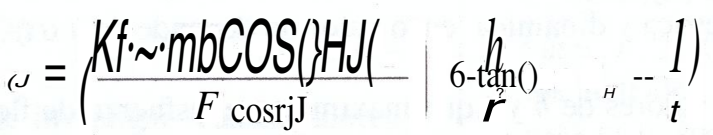

Para una geometría dada de un diente de engrane, existirá solo una posición definida del ángulo [] (ver figura NO.3), que corresponderá al valor máximo del esfuerzo. Esta posición podría hallarse matemáticamente por el teorema de máximos y mínimos si se logra expresar ( $J$ en función exclusiva del ángulo de posición []. Con ayuda de la figura No.3, se observa que:

\section{$h=\overline{O Q}-\overline{O G}$}

donde: 
$O Q=\frac{O E}{\operatorname{sen} O_{H}}=\frac{r_{b}}{\operatorname{sen} O H}$

$\mathrm{OG}=01-$ ACsene $={ }^{\prime} A \cos f 3-r($ sene

$r_{A}=r_{D}+r_{f}$

Por lo tanto:

$h=\frac{r_{b}}{\operatorname{sen} \theta_{H}}-r_{A} \cos \beta_{A}+r_{f} \operatorname{sen} \varepsilon$

Por otro lado, el semigrosor basal del diente es :

$\stackrel{t}{\underline{z}}=A l-A C$ cose $=r A \operatorname{senf} 3 A-r f$ cose

$t=2\left(\operatorname{rasenf} 3_{A},>, \operatorname{cose}\right)$

El ángulo $f 3_{A}$ depende de la fonna del perfil del flanco del diente y se calculará luego.

El esfuerzo en la raíz del diente en un punto genérico puede expresarse, en virtud de la ecuación NO.18 como:

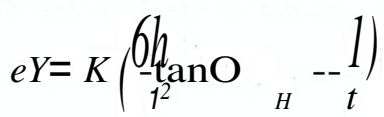

donde $K$ es una constante geométrica y dinámica cuyo valor no $\sim$ epende de $h$ o $t$.

La sección critica corresponde a valores de $h$ y $t$ que maximizan el esfuerzo de flexión, por lo tanto, aplicando el principio del máximo de una función de dos variables:

$d e Y=-\frac{\delta h}{c m}+d t \quad \begin{array}{cc}i h \\ O t\end{array}=0$

Derivando la función $e Y$ e igualándola a cero:

$K\left(\frac{6}{t^{2}} \tan \theta_{H}\right) d h+K\left(\frac{-12 h \tan \theta_{H}}{t^{3}}+\frac{1}{t^{2}}\right) d t=0$

Despejando el gradiente $d h / d t$ y simplificando, se obtiene: 


\section{$\frac{d h}{d t}=-\frac{2 h}{t}-\frac{1}{6 \tan B_{H}}$}

Ahora con ayuda de las ecuaciones NO.20 y 21 , se expresa el gradiente $d h / d t$ como:

$$
\frac{d h}{d I}=\frac{d h / d e}{d I / d e}=\frac{\underline{r} i \underline{\text { cose }}}{2 r_{i s e n e}}=\frac{1}{2 \text { tane }}
$$

Igualando las ecuaciones NO.22 y 23 :

$$
\frac{2 h}{t} \quad 6 \frac{1}{\tan B H}=\frac{1}{2 \operatorname{tane}}
$$

Sustituyendo $h \mathrm{Y} t$ por las ecuaciones NO.20 y 21 respectivamente en la ecuación anterior, se logra la siguiente ecuación trascendental para calcular el ángulo 8 :

$$
\text { cote }=2 \frac{\left((r b / \operatorname{sen} B H)-r A \quad \operatorname{COSPA}+r_{i} \text { sene }\right)}{r A \operatorname{sen} P A-r_{f} . \operatorname{cose}}-\frac{1}{3 \tan B H}
$$

Esta ecuación puede ser que resulte para el ángulo 8 por algún método numérico apropiado.

Por inspección de la figura No.3, es obvio que la solución debe estar comprendida en el ámbito $0^{\circ} \sim \mathrm{e} \sim 90^{\circ}$

Con el propósito de investigar cuáles valores del ángulo 8 se consideran típicos, se resolvió la ecuación

NO.21 por medio de un programa de computadora para diversas combinaciones de $\mathrm{N}(20,30,60,1000)$ Y Nc $(20,30,60,1000)$, encontrándose que todas las soluciones quedaban comprendidas entre $\mathrm{e}=20$ 。 y $\mathrm{e}=35^{\circ}$.

Igualmente se comprobó que la función de esfuerzo, dada por la ecuación NO.18, es poco sensible a la variación del ángulo 8 dentro del intervalo citado. Por esta razón, para fines prácticos se asume que la sección critica se localiza en un valor e $=30^{\circ}$ para todos los casos, independientemente de los valores de los demás parámetros . Consecuentemente, en el modelo matemático propuesto se fijará el valor $\mathrm{e}=30$. Este resultado concuerda con los hallados por investigadores japoneses al usar el método de elementos finitos (Tanaka, Kohida, Yamada, 1986 ).

\section{CÁLCULO DE PA PARA DIENTES CON TRANSICIÓN DE PERFIL POR ENCIMA DEL CÍRCULO BASE.}

En la figura NO.4 se ilustra este tipo de situación donde el perfil del diente es de evolvente en toda su extensión por arriba del arco del filete o entalle de raíz. Esta situación ocurre cuando la geometría del diente es tal que el punto de transición $\mathrm{T}$, entre el arco de enlace DT y la curva de evolvente, ocurre por 
encima del CÍrculobase. Matemáticamente lo anterior puede establecerse como $\operatorname{rr}$ 2: $r_{b}$. siendo $r r$ la coordenada radial del punto $\mathrm{T}$.

$\beta_{A}=\varphi_{A}-\gamma_{T}$

Analizando el triángulo OAC, se ve que:

$\varphi_{A}=\operatorname{arcos}\left(\frac{r_{b}}{r_{A}}\right)$

Por relaciones de la involumetria:

$\left.r r=f J \cdot \frac{90}{N-(\operatorname{tanfj} \mathbf{J}}-\tan \sim \mathbf{r}\right)$

Pero a su vez, analizando el triángulo OCT:

$\tan \varphi_{T}=\sqrt{\left(\frac{r_{A}}{r_{b}}\right)^{2}-1}-\frac{r_{f}}{r_{b}}$

Combinando las ecuaciones No.25, 26, 27, Y28, se resuelve para $P_{A^{\prime}}$ obteniéndose:

$$
\beta_{A}=\frac{90}{N}-\phi+\operatorname{arcos}\left(\frac{r_{b}}{r_{A}}\right)+\frac{180}{\pi}\left(\tan \phi+\frac{r_{f}}{r_{b}}-\sqrt{\left(\frac{r_{A}}{r_{b}}\right)^{2}-1}\right)
$$

\section{CÁlculo de PA PARa dientes CON TRANSICIÓN DE PERFIL POR DEBAJO DEL CÍRCULO BASE.}

Cuando el CÍrculo de dedendo es menor al círculo base, el perfil del diente experimenta una transición en el punto que alcanza al CÍrculo base. Por encima del CÍrculobase, el perfil es la involuta de este CÍrculo; sin embargo por debajo del CÍrculobase, el perfil del flanco quedará definido por la fonna y la

Por ahora se procederá a calcular el ángulo $P A^{\prime}$ Por inspección de la figura No.4, se observa que: 
son un poco más resistentes al esfuerzo de flexión. Por lo tanto, el uso de este modelo de flanco radial rectilíneo, producirá valores de J especialmente adecuados para engranes de conformación, en tanto que su uso para engranes de generación, producirá resultados conservadores, lo cual no resta validez o utilidad al modelo como herramienta segura de diseĺlo.

Para calcular el ángulo $f 3_{A}$ mostrado en la figura No.5, se procede de la manera siguiente:

$f J A=f 3 s+r$

donde:

$\beta_{B}=\frac{t_{b}}{2 r_{b}} ; t_{b}=2 r_{b}\left(\frac{\pi}{2 N}+i n v \phi-i n v \varphi_{B}\right) ; i n v \varphi_{B}=\varphi_{B}=0 ; i n \nu \phi=\tan \phi-\phi$

Así:

$\beta_{B}=\frac{\pi}{2 N}+\tan \phi-\phi$

Trabajando en cl triángulo OAT:

$\operatorname{seny}=\frac{A l^{\prime}}{O A}=-{ }^{r c}$

$r=a /-\operatorname{csen}\left(\begin{array}{c}\left.[]_{1}\right] \\ r_{A}\end{array}\right.$

Sustituyendo las ecuaciones NO.31 y 32 en la 30 :

$f J A=-\underset{2 N}{-7 r}+\tan t j J \cdot\left[j J+\operatorname{arcsen}\left(\underset{r_{A}}{r_{-}}\right]\right.$

en radianes.

Finalmente. convirtiendo este valor a grados:

$f J A=\frac{90}{N}+-_{7 r}^{180} \tan \left[j J \cdot+j J+\operatorname{arcsen}\left(\underset{r_{A}}{-L}\right)\right.$ 


\section{CONDICIONES DE TRANSICIÓN ENTRE UN PERFIL SIN FLANCO RECTILÍNEO y UNO CON FLANCO RECTILÍNEO.}

La condición de transición ocurre cuando en la figura NO.5 el radio del punto de transición T, coincide con el radio base, es decir: $r r=r_{b}$ Habrá flanco rectilíneo solamente si $r r \quad:, r_{b}$ Este criterio será útil para saber si el ángulo $f 3_{A}$ deberá calcularse con la ecuación NO.29 (sin flanco rectilíneo), o con la ecuación NO.33 ( con flanco rectilíneo ).

En el triángulo OAT de la figura No.5, se observa que :

$$
r_{T}=O T=\sqrt{r_{A}^{2}-r_{f}^{2}}
$$

Considerando que $r_{\boldsymbol{i}} \ll<r_{A^{\prime}}$ para fines prácticos se tomará $r r=r_{A}$.

Ahora será fácil escoger la ecuación correcta para calcular $f 3_{A}$

Usar ecuación NO.29 si $r_{4}>r_{b}$.

Usar ecuación NO.33 si $r_{A}<r_{b}$

$$
K_{t}=H+\left(\frac{t}{r_{f}}\right)^{L}\left(\frac{t}{h}\right)^{M}
$$

donde: $\quad H=0.340-0.4583662 \sim$

$$
\begin{gathered}
L=0.316-0.458366 \sim \\
M=0.290+0.4583662 \sim
\end{gathered}
$$

$r p=$ ángulo de presión nominal en radianes.
En caso de igualdad $\left(r_{A}=r_{b}\right)$, cualquiera de las ecuaciones deberá producir el mismo resultado.

\section{FACTOR DE CONCENTRACIÓN DE ESFUERZOSPORFATIGA $K_{i}$}

El factor $K_{\mathbf{i}}$ depende del factor teórico o geométrico de concentración de esfuerzos $\mathrm{Kt}$ y de la sensibilidad a la entalla $q$, según la relación:

$$
K_{f}=1+q\left(K_{t}-1\right)
$$

Como una medida conservadora y por simplicidad, se asumirá un valor unitario para $q$; con lo cual $K_{i}=K_{t}$

En la literatura consultada ( Deutschman, AD., 1985, p.577; Mitchiner, RG. y Mabie. H. H.,1980 ; Peterson RE., 1974; Shigley,J. y Mitchell, L., 1985, p. 635 ), aparecen diversas fónnulas empíricas para la detenninación del factor $\boldsymbol{K}_{t}$. Haciendo una síntesis de estas fonnulaciones, se propone calcular el $K_{t}$ con la ecuación siguiente:

$r_{\mathbf{i}}=$ radio del filete en la raíz del diente(ver ecuaciones NO.11 y 12 ).

\section{RELACIÓN DE COMPARTICIÓNDE CARGAmN.}

La relación de compartición de carga sedefine como la razón entre la carga transmitida por una pareja de dientes en un instante dadoy la 
carga total que se transmite a lo largo de la línea de transmisión. Consecuentemente el valor de $\mathrm{mN}$ es inferior a la unidad mientras exista algún grado de compartición de carga entre dos o más parejas de dientes. En el caso de los engranes rectos estandarizados, solo existe compartición de carga durante parte del ciclo de contacto, dándose una parte del ciclo sin ninguna compartición de carga, el cual se inicia precisamente en el punto más alto de contacto ( fase o posición de máximo esfuerzo flector ). Dada la definición del factor $\mathrm{mN}$, este tendrá valor tUlítario en la fase del pmIto más alto de contacto para el caso de los engranes rectos. Es decir que se utilizará $m N$ en el cálculo del factor $\mathrm{J}$ respectivo.

\section{COMPROBACIÓN DEL MODELO MATEMÁTICO.}

Con el propósito de probar el funcionamiento del modelo matemático y verificar la validez de sus resultados, se decidió generar datos para graficar curvas del factor $\mathrm{J}$ en función del número de dientes del engrane y compararlas con las curvas normalizadas de la AGMA.

Para efectos de esta comparación, se escogió trabajar con un sistema de dientes estandarizado de altura completa con las siguientes $\quad$ especificaciones: $\quad r j J=20$, $K_{a}=1, \quad K_{b}=1.4, \quad K_{r}=0.35$ para generación y $K_{r}=0.30$ para conformación.

Con el fin de cubrir valores extremos y medios de la familia de curvas AGMA, se decidió generar por medio del modelo matemático, únicamente las curvas para los siguientes valores del número de dientes del engrane compallero: $\quad N_{\mathrm{e}}=17, \quad N_{c}=50 \quad$ y $N_{c}=1000$.

Programando el modelo matemático en una hoja electrónica, se calcularon los valores del factor J para los siguientes valores del número de dientes del engrane principal $N: 20,30$, $60,80,100,200,300$ Y 500.

Los resultados se muestran en las figuras NO.6, 7 y 8 que contienen las gráficas de los factores $J$ en función del número de dientes del engrane analizado $\quad N$ para los datos anteriores. Analizando estas curvas, se pueden sintetizar las siguientes observaciones:

- Para engranes cortados por generación, el modelo matemático produce valores del factor J muy similares a los valores estandarizados de la AGMA. siendo las curvas prácticamente coincidentes para $N$ :2: 100 dientes y existiendo una ligera desviación entre las curvas para $N<100$ dientes.

- Para fines de disello, la desviación del modelo matemático con respecto a la curva AGMA para engranes de generación, es relativamente pequella (inferior al $10 \%$ en el peor caso) y benévola en todo caso, ya que esta desviación produciría un ligero sobredisello del engrane.

- Para engranes cortados por conformación, el modelo matemático produce una curva del factor $J$ de forma similar a la de engranes cortados por generación, pero de valores notoriamente inferiores, lo cual no debe interpretarse como un error del modelo, sino como un resultado fiel a la realidad de que los engranes cOlúormados tienen dientes geométricamente diferentes y más débiles a la flexión que los dientes de los engranes de generación. Desafortunadamente, la normalización AGMA no incluye curvas particulares del factor $J$ para engranes de conformación y tampoco se lograron encontrar en la literatura consultada valores publicados del factor $J$ para engranes de conformación que sirvieran de referencia o comparación. 


\section{CONCLUSIONES}

Con base en los resultados obtenidos, se pueden derivar las conclusiones siguientes:

- El modelo matemático desarrollado aproxima fielmente a las curvas del factor $\mathrm{J}$ de la AGMA para engranes de generación, existiendo leves desviaciones cuando el engrane tiene menos de 100 dientes; las cuales son benévolas para efectos de un disello conservador.

- Las desviaciones con respecto a los valores AGMA se atribuyen principalmente a la fonna de modelar el arco de la raíz del diente creado por una cremallera de generación. La forma real y exacta de este arco corresponde a una curva conocida como " trocoide ", la cual fue simulada o aproximada mediante un arco circular en el modelo matemático. Por otro lado, de acuerdo con Mitchiner y Mabie (Transactions of the ASME, Vol. 104, January 1982), los factores $J$ de la AGMA fueron obtenidos empleando técnicas gráficas, posiblemente dibujando los perfiles exactos de los dientes por medio de involutómetros de cremallera.

- El modelo matemático desarrollado es particulannente útil para calcular el factor $J$ de los engranes cortados por fresas de confonnación. En este caso el arco de raíz del diente de confonnaCÍón es realmente un arco circular, que corresponde exactamente al arco circular utilizado por el modelo matemático, de tal fonna que no se introduce error alguno en este proceso. Debido a la total ausencia de valores del factor $J$ para engranes de confonnación en la literatura, es práctica común de diseño asumir que dichos factores $\mathbf{J}$ son iguales a los valores AGMA para engranes de generación. Esta práctica conduce a un diseño inseguro del engrane y debe evitarse. Se considera que el modelo matemático aquí propuesto, significa un aporte de singular importancia como herramienta de cálculo para el disello correcto de engranes fabricados por confornlación, los cuales representan la mayoría de los engranes producidos enlos países en desarrollo, incluyendo a Costa Rica.

- Se encontró que para localizar en forma exacta la sección critica a la flexión enla base del diente, se debe resolver una compleja ecuación trascendental para el ángulo i:; por medio de técnicas numéricas. Estrictamente, el ángulo i:; tendrá un valor particular para cada conjunto de valoresde $N, N$ e, rjJ. Sin embargo, se encontró que para efectos prácticos, el modelo matemático funciona razonablemente bien cuando se fija la sección critica en la posición de $[;=30$.

\section{BIBLIOGRAFIA}

1. Casillas, AL. Máquinas. Edición 34. Edición Hispanoamericana. MELSA España, 1988.

2. Deutschman, Aaron D. et al. Diseño de Máquinas. Segunda edición. CECSA México, 1987.

3. Dudley, Darle W. Manual de engranes. Compailia Editorial Continental S.A México, 1973.

4. Faires, V. M. Diseño de Elementos de Afáquinas. Montaner y Simon, S.A. Espalla, 1970.

5. Giesecke, Frederick E. et al. Dibujo Técnico. Sexta edición. Limusa. México, 1989.

6. Hall, A S. et al. Teoría y Problemas de Diseño de Afáquinas. McGraw-Hill. México, 1971.

7. Mitchiner, R. G. et. al., The determination of the Lewis Form Factor and the AGM4 Geometry

8. Factor J for External Spur Gear Teeth. Transactions of the ASME, Vol. 104: 148 - 158, Jan. 1982. 
9. Pacheco, A. et. al., lvfodelo matemático para calcular el factor geométrico $J$ en engranes rectos. Ingeniería 6 (1) : 81-96, 1996, San José, Costa Rica.

10. Peterson, RE. Stress Concentration Design Factors. John Wiley. 1974.

11. Shigley, Joseph y Charles Mischke. Diseño en Ingeniería Mecánica. Quinta edición. McGraw-Híll Interamericana S.A. México, 1990.
12. Shigley, Joseph y Larry Mitchell. Diseño en Ingeniería Afecánica. Cuarta edición. McGraw-Hill. México, 1985.

13. Shigley, Joseph y Jo1m Uiker. Teoría de Afáquinas y Afecanismos. McGraw-Híll Interamericana S.A. México, 1983.

14. Tanaka, M. ; Kohida H. and Yamada A. Stress Ana yses of Spur Gears in Consideration ofSpecific Sliding. Bulletín of JSME, Vol. 29, No. 250, April 1986. p. $1319-1325$. 


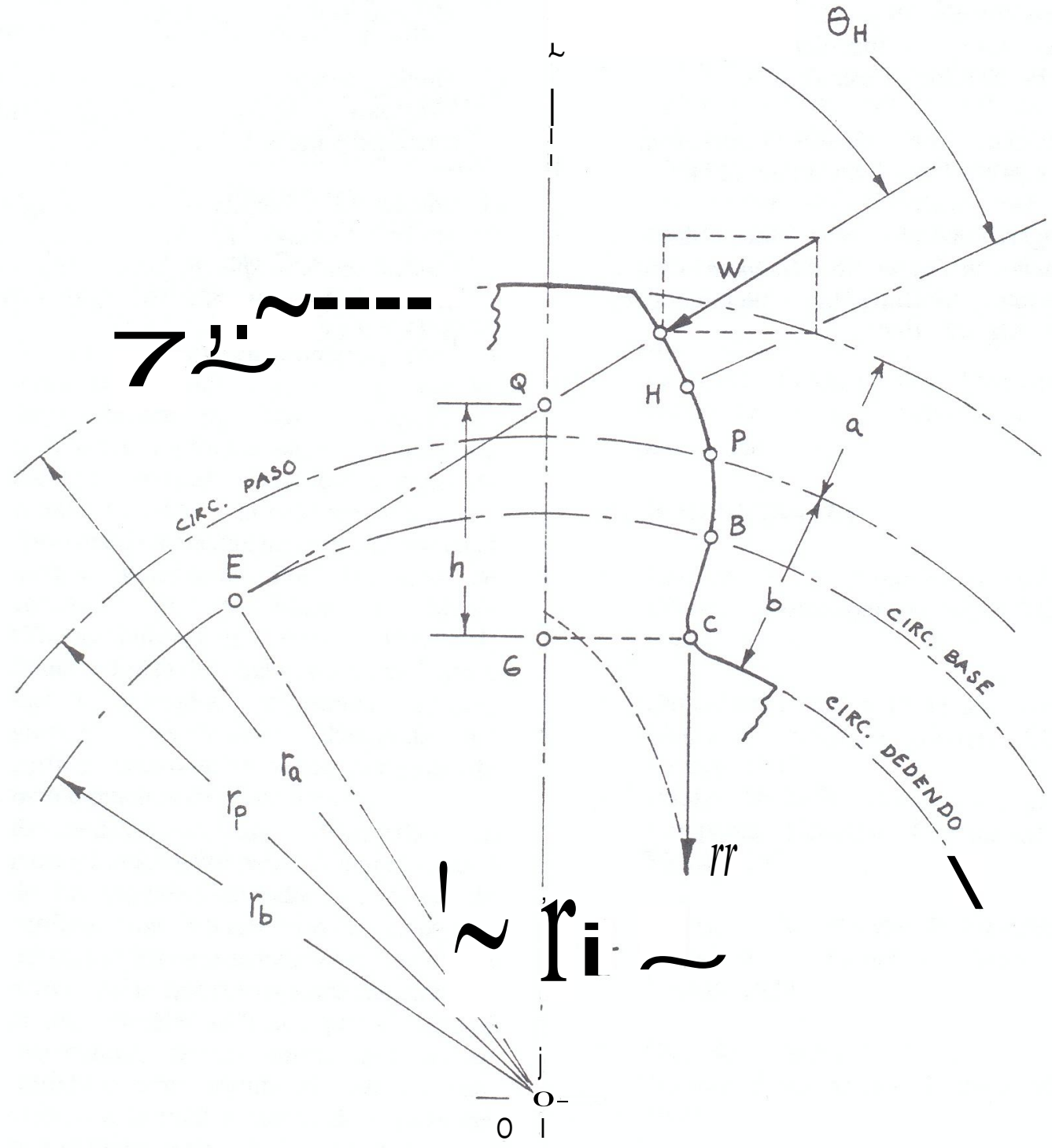

Figura 1.Diente de engrane recto con perfil de evolvente. Parámetros geométricos básicos. 
PACHECO: Método analítico para calcular el factor ...

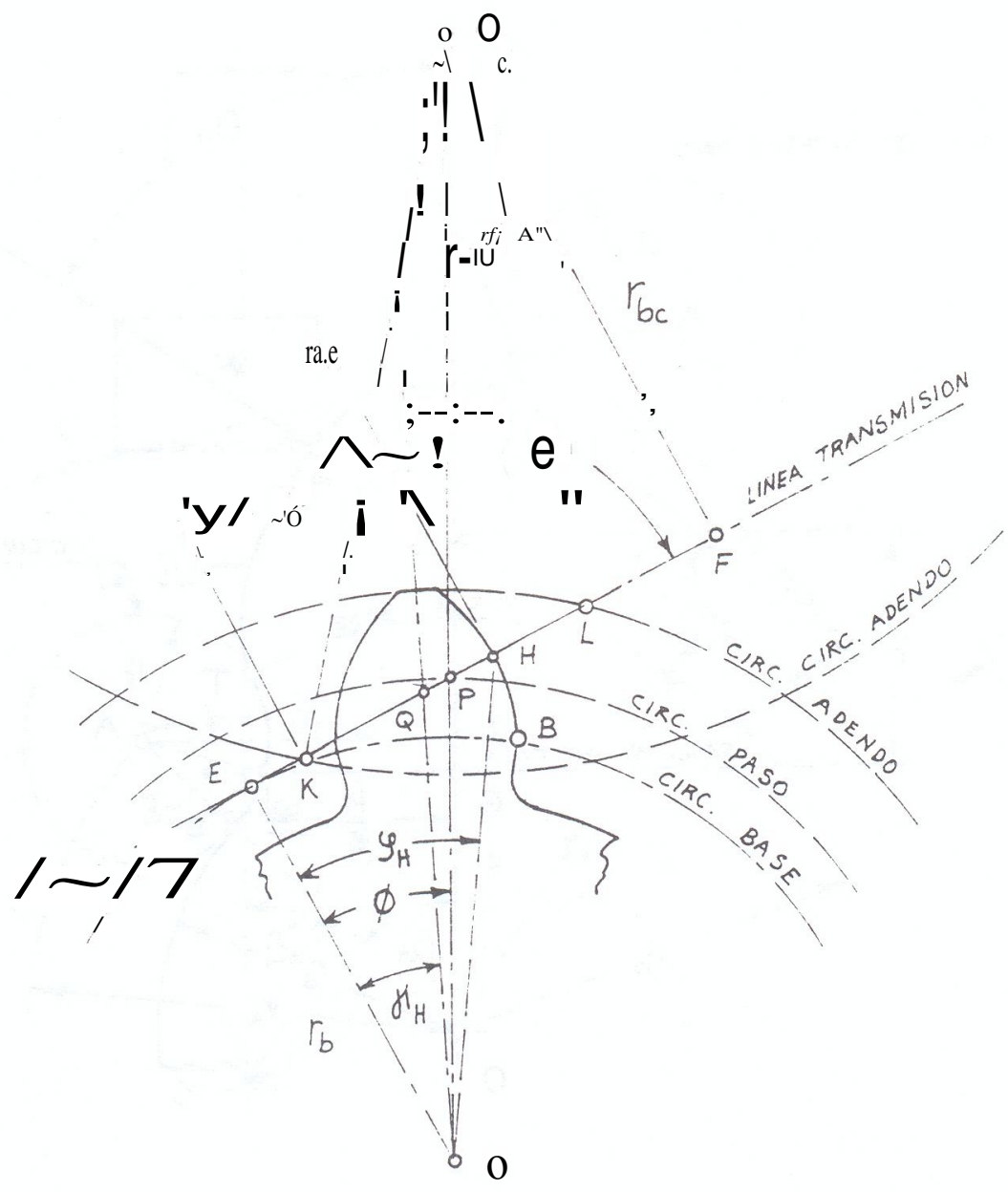

Figura 2. Determinación del ángulo de presión relativo eH para el punto más alto de contacto. 


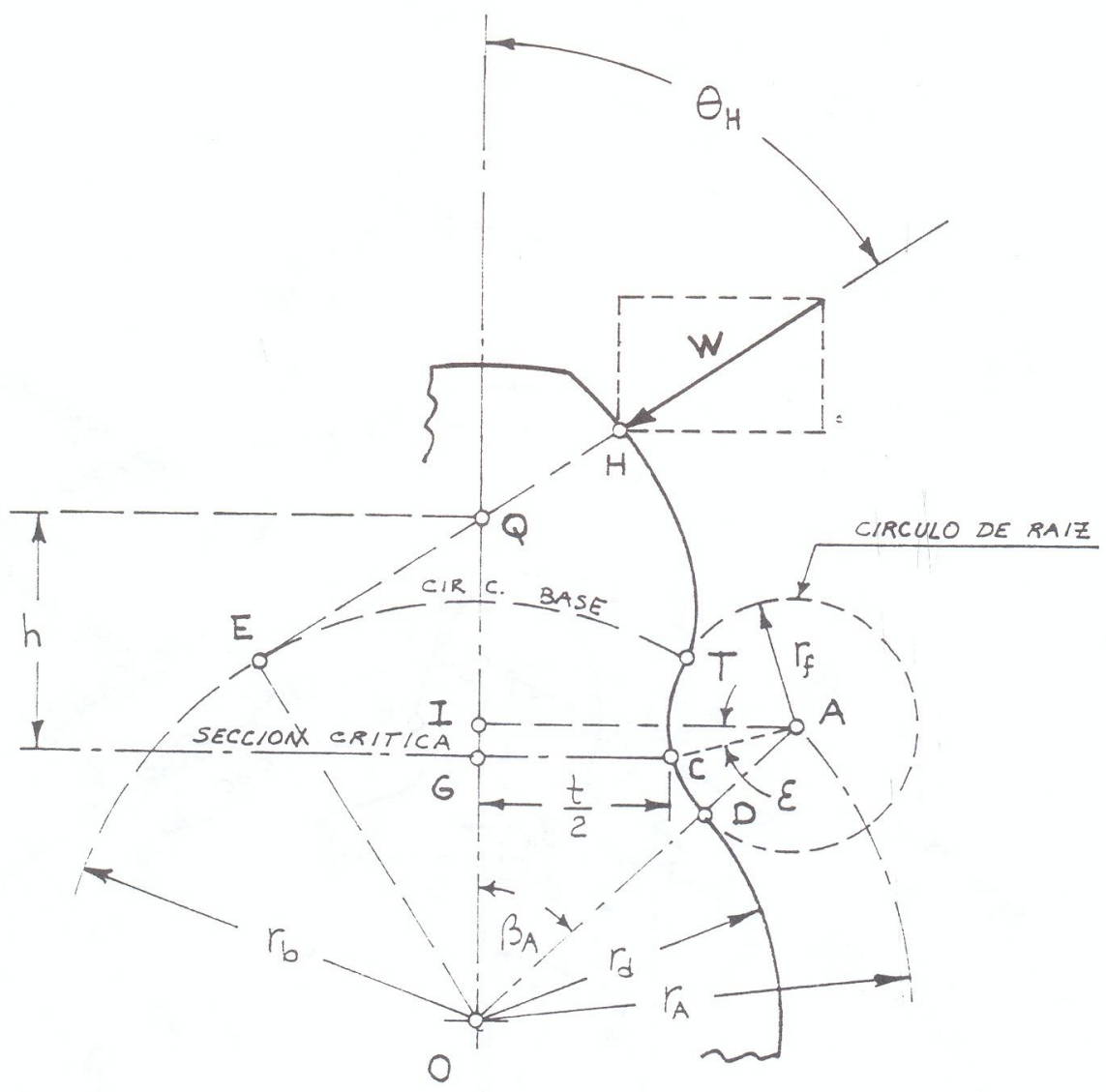

Figura 3. Determinación de la sección crítica. Valores de h y t. 


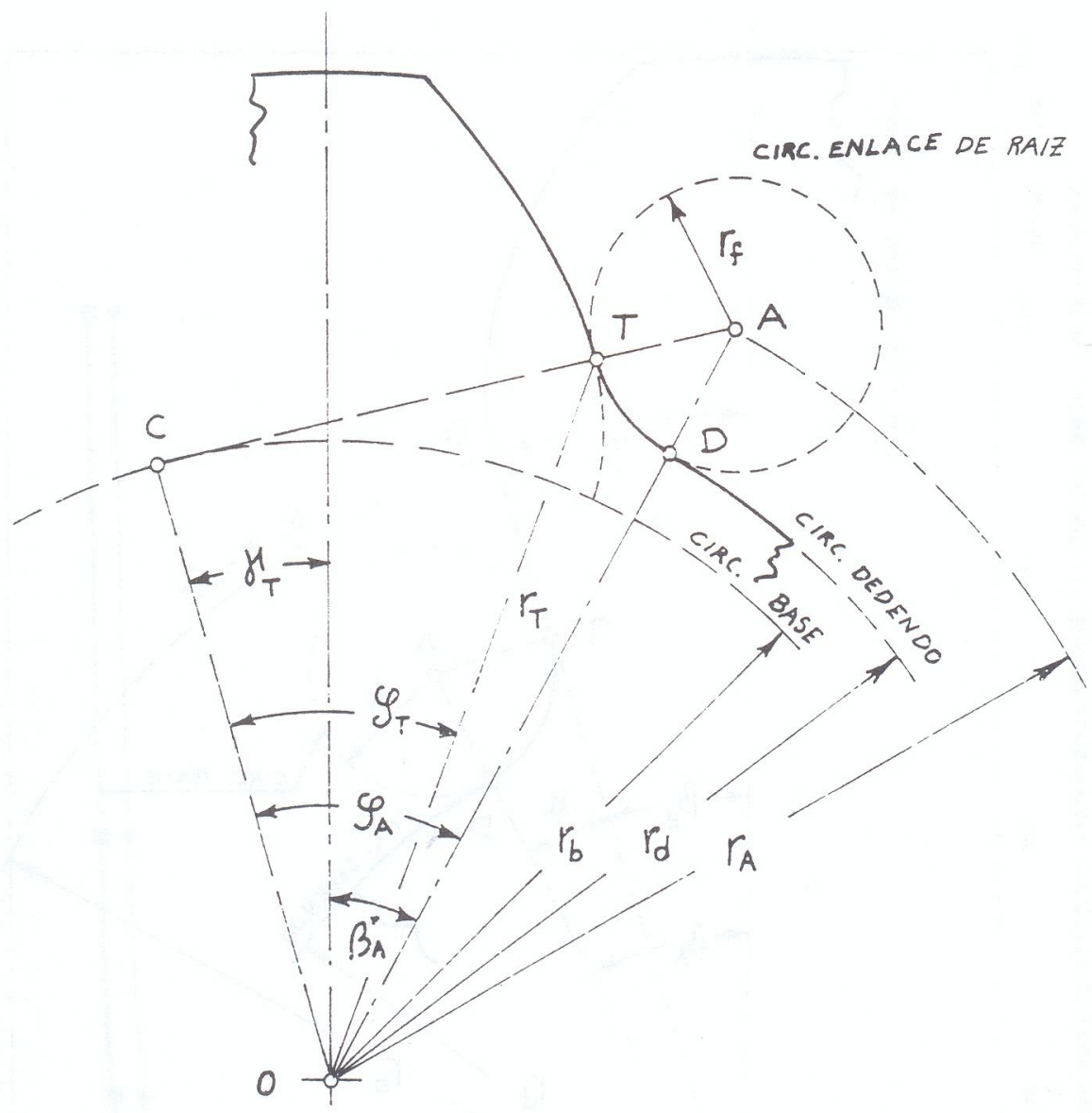

Figura 4. Diente de evolvente con transición de raíz por encima del círculo base. 


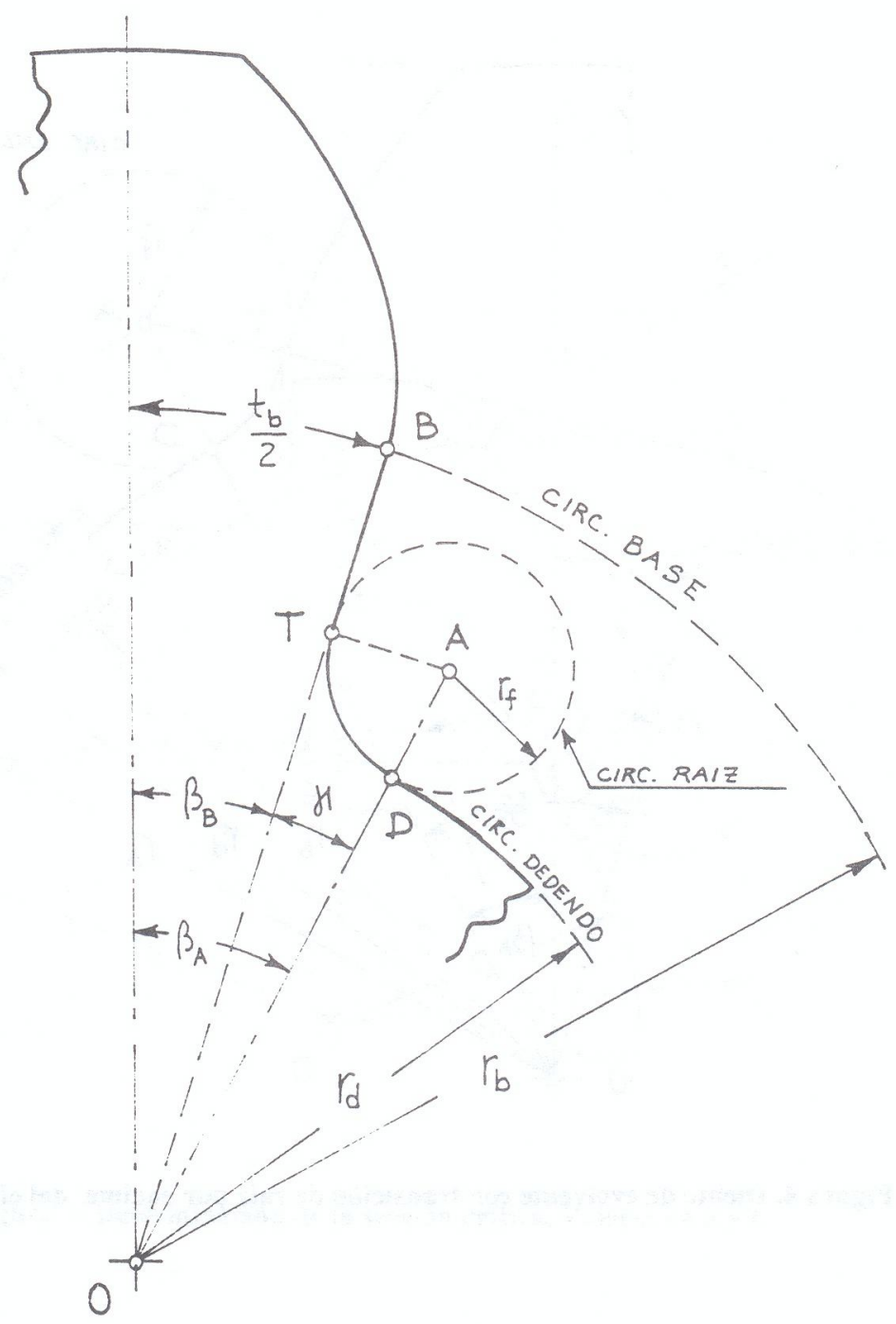

Figura 5. Diente de evolvente con traDllición de raíz por debajo del círculo base. 


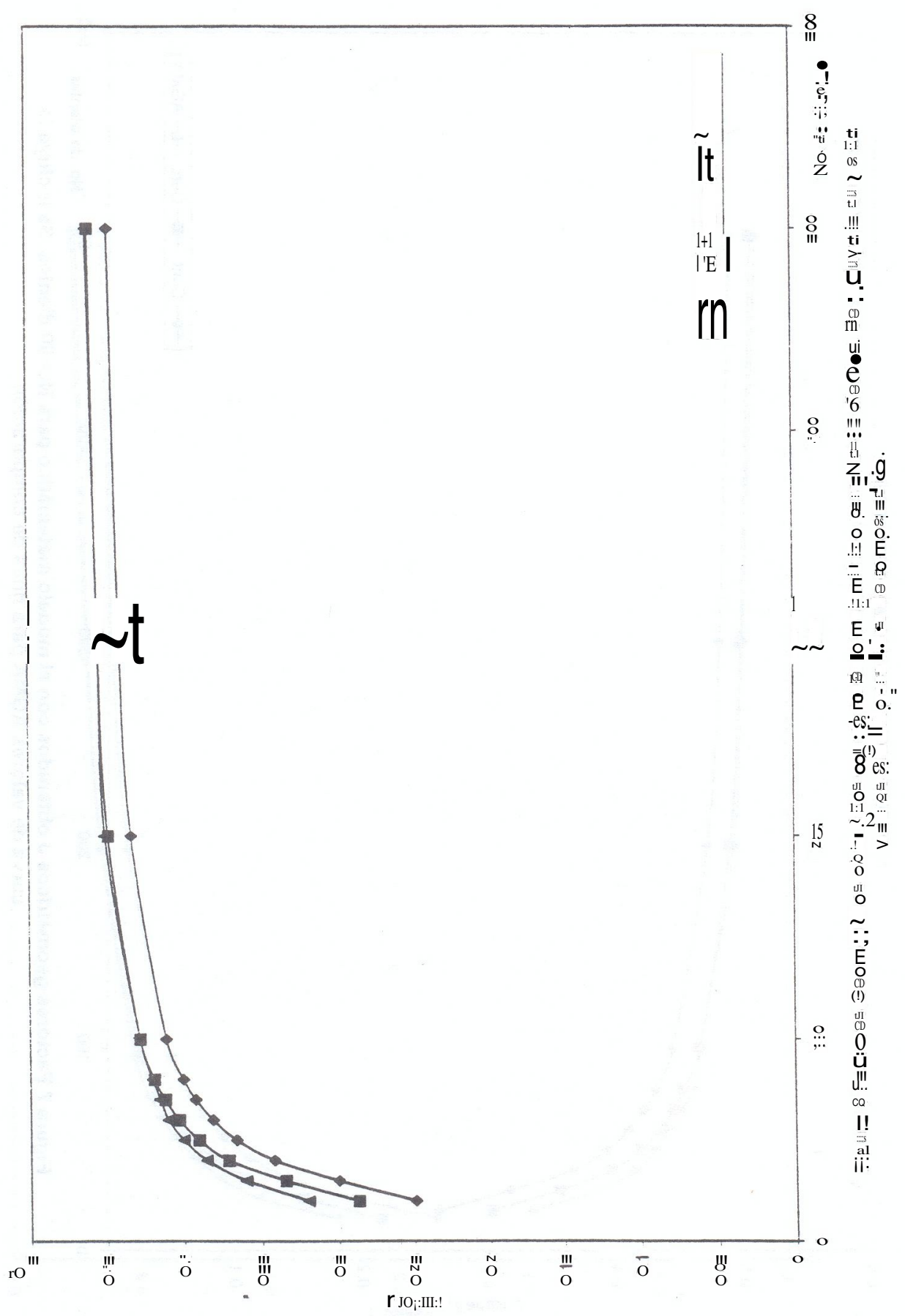




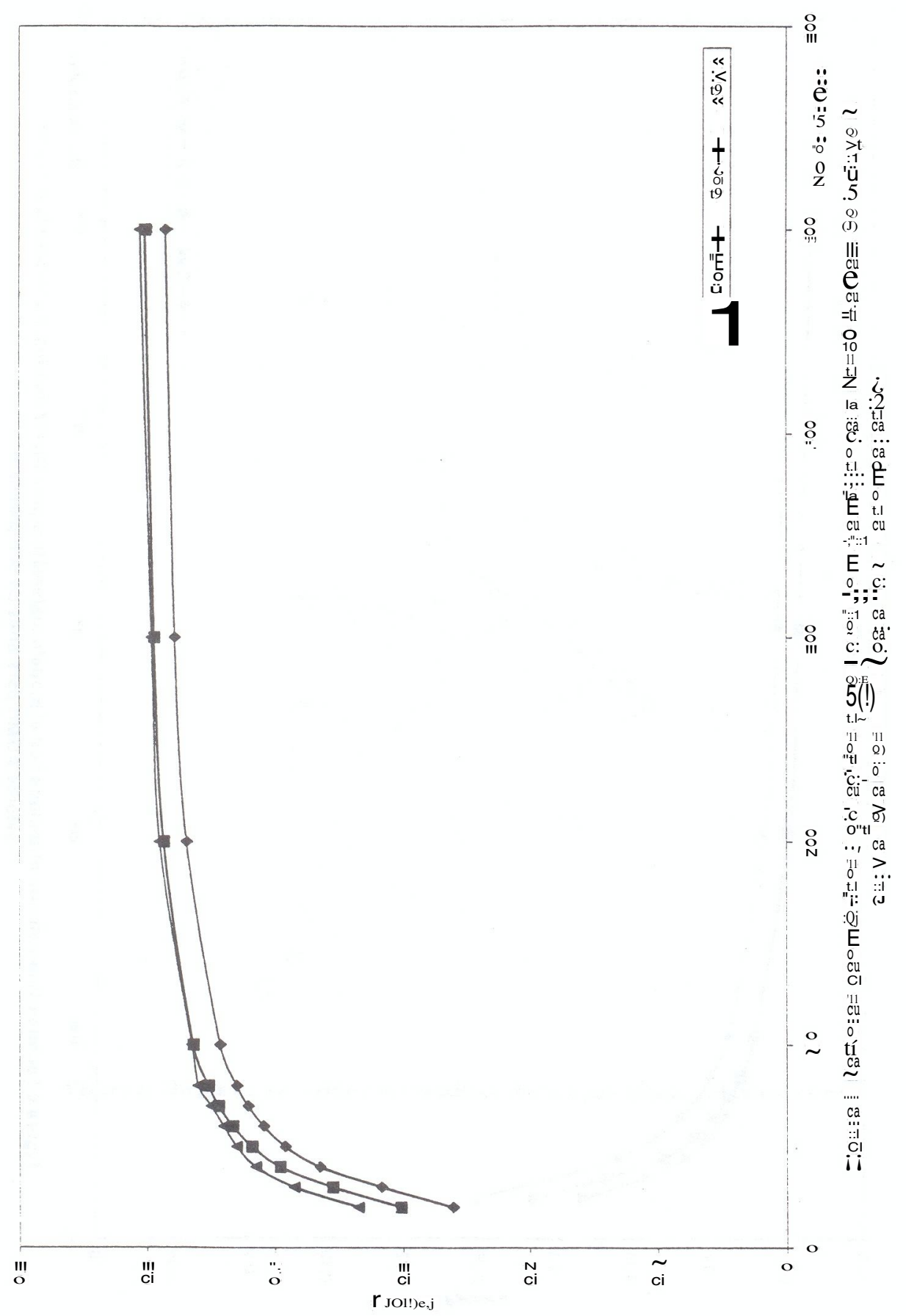


PACHECO: Método analítico para calcular el factor ...

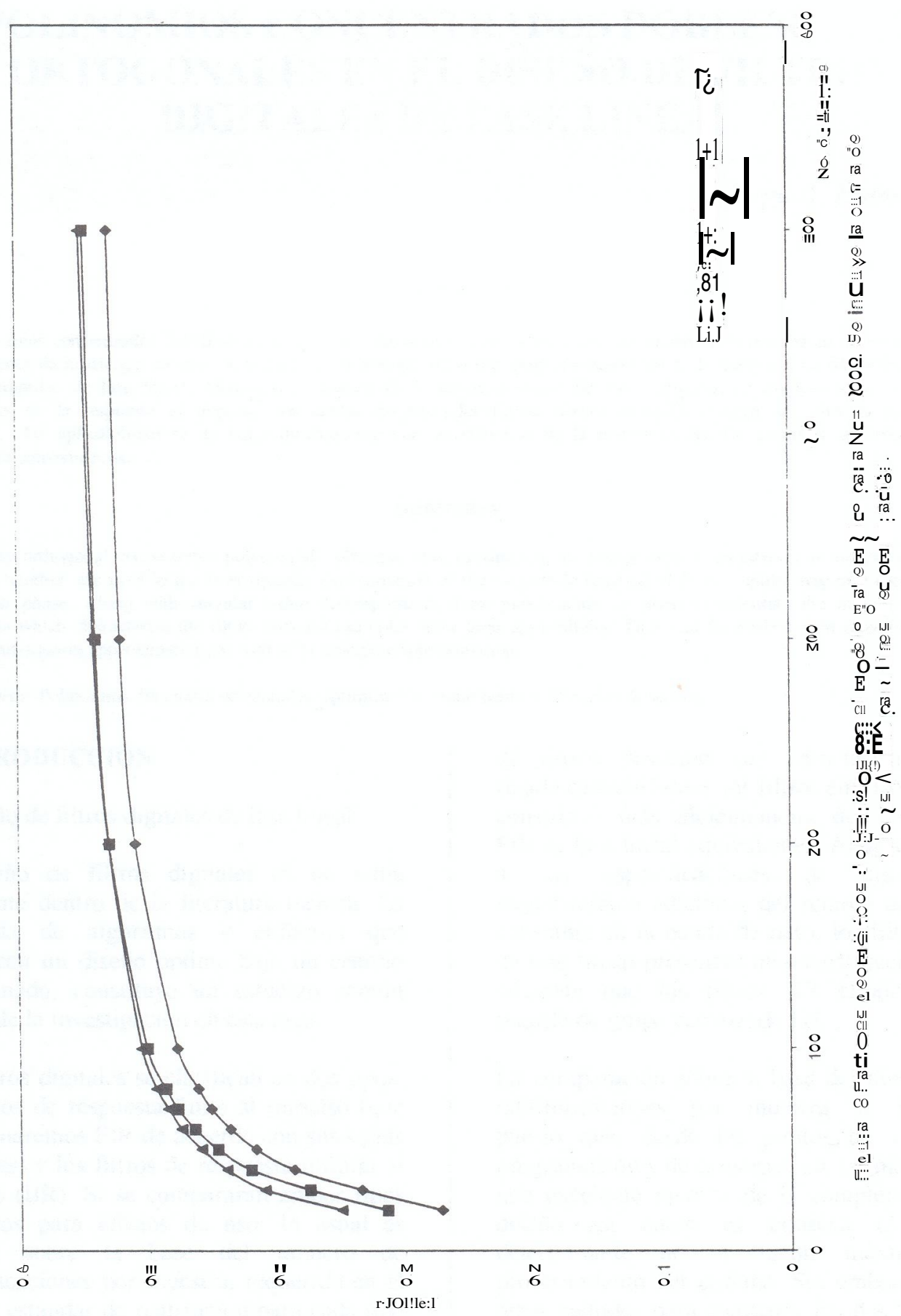

